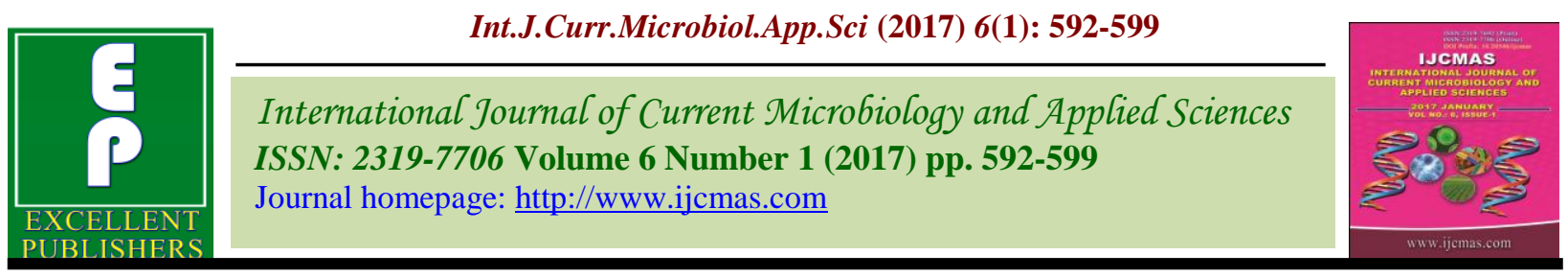

Original Research Article

http://dx.doi.org/10.20546/ijcmas.2017.601.072

\title{
Vascular Catheter Related Infections in a Tertiary Care Hospital - A Microbiological Study
}

\author{
M.V.M. Sainivedeta, I.M. Rejitha*, G. Sucilathangam and C. Revathy \\ Department of Microbiology, Tirunelveli Medical College, Tirunelveli - 627 011, \\ Tamil Nadu, India \\ *Corresponding author
}

\section{A B S T R A C T}

\section{Keywords \\ Catheter-related bloodstream infection (CRBSI), catheter colonisation, catheter site (local) infection, Intravenous catheter, Catheter days.}

Article Info

Accepted:

29 December 2016 Available Online: 10 January 2017
Catheter-related bloodstream infection (CRBSI) is defined as the presence of bacteraemia originating from an intravenous catheter. It is one of the most frequent, lethal, and costly complications of central venous catheterization and also the most common cause of nosocomial bacteraemia. Hence the present study was undertaken to find the incidence of catheter colonisation, catheter site (local) infection and CRBSI among patients with indwelling vascular catheters in a tertiary care hospital and determine their microbiological profile. This was a cross-sectional observational study included 50 patients with an indwelling vascular catheter in Tirunelveli Medical college hospital for the period of 6 months. Catheter tips and Blood samples were collected and subjected into Culture and Antibiotic sensitivity testing. Out of the 50 patients studied, 34 isolates were obtained from the catheter tips and blood cultures. The majority of them were Coagulase negative staphylococci $(44.1 \%)$ followed by Staphylococcus aureus(17.6\%) and Klebsiella pneumoniae (17.6\%). Diphtheroids were $14.7 \%$ and Pseudomonas sp were $5.9 \%$ respectively. The incidence of CRBSI in this study was found to be 1.38 per 1000 catheter days. Of the 5 isolates that caused CRBSI, 2 were MRCONS,2 were MRSA, and one was an ESBL producing Klebsiella pneumoniae. An estimation of the rate of infection, and identification of the causative organisms will provide a clue as to the route of entry and pathogenesis of the infection, and this in turn may point to effective preventive strategies.

\section{Introduction}

Catheter-related bloodstream infection (CRBSI) is defined as the presence of bacteraemia originating from an intravenous catheter. Some $20-30 \%$ of all nosocomial bacteraemia occur in the ICU, with an incidence rate that ranges from 2.5 to 6.7 episodes per 100 admissions. The majority of serious CRBSI up to $87 \%$ are associated with central venous catheters (CVC) (Vincent et al., 1995). Patients may be colonized with hospital-acquired organisms and venous access might be needed for extended periods of time. The catheter may be manipulated multiple times per day for the administration of fluids, drugs, and blood products. 
CRBSI arise from any of four major sources: skin colonization, intraluminal or hub contamination, secondary seeding from a blood stream infection and rarely, contamination of the infusate (Anaissie et al., 1995; Bjornson, 1993). The most common source of CRBSI is colonization of the intracutaneous and intravascular portions of the catheter by microorganisms from the patient's skin and occasionally the hands of health care workers (Bjornson et al., 1982). Thus, common skin commensals, such as coagulase-negative staphylococci and Staphylococcus aureus, are often isolated from colonized catheters and patients with primary CRBSI. Administration of contaminated infusate or additives such as contaminated heparin flush can result in a BSI although this is now rare.

Catheter colonisation is characterised by significant growth of a microorganism on the catheter but no growth in blood cultures. Local site infection is indicated by the presence of erythema, swelling, tenderness, and purulent drainage around the catheter port that grows a microorganism on culture. Blood cultures may be positive or negative. CRBSI is often suspected clinically in a patient who presents with fever or chills, unexplained hypotension, and no other localizing sign. Mild symptoms include malaise and nausea, and severe symptoms include high fever with rigors, hypotension, vomiting etc.

The diagnosis of CRBSI requires a positive culture of blood from a peripheral vein and clear evidence that the catheter is the source. CRBSI means a patient with an intravascular catheter has at least one positive blood culture obtained from a peripheral vein, clinical manifestations of infections (i.e., fever, chills, and/or hypotension), and no apparent source for the BSI, except the catheter. In addition, one of the following is also present: A positive semi-quantitative ( $>15 \mathrm{CFU} /$ catheter segment) or quantitative $\left(>10^{3} \mathrm{CFU} / \mathrm{ml}\right.$ by catheter flush method) catheter tip culture also, the same organism (species and antibiogram) is isolated from the catheter segment and peripheral blood culture. Hence this study was undertaken to find the incidence of catheter colonisation, catheter site (local) infection and CRBSI among patients with indwelling vascular catheters in a tertiary care hospital and determine their microbiological profile.

\section{Materials and Methods}

This was a cross-sectional observational study included 50 patients with an indwelling vascular catheter in Tirunelveli Medical college hospital for the period of 6 months. Patients above 12 years of age and either sex were included in this study. Patients with sepsis at the time of admission were excluded from the study.The presenting symptoms and risk factors such as duration of hospital stay, duration of catheter in situ, site of placement of catheter, diabetes, renal failure, immune suppression etc was identified and noted in the proforma.

\section{Sample collection and processing}

\section{Catheter tip sampling and processing}

The skin was cleaned with $70 \%$ alcohol prior to catheter removal. The catheter was held at the proximal end and carefully removed from the patient with a sterile instrument, taking care to avoid contact with the skin. The distal end was held over a sterile tube, and the tip was cut with sterile scissors. A $5 \mathrm{~cm}$ segment from the distal tip of the catheter was collected in the tube and transported to the lab as soon as possible. Extra luminal Maki's roll over plate method and endo luminal catheter flush culture was used for processing. Extra luminal Maki's roll over plate method: Using sterile forceps, the catheter tip was rolled 
back and forth across the entire surface of a blood agar plate using sterile forceps. For endo luminal catheter flush culture, the catheter lumen was flushed into a sterile vial with $1 \mathrm{ml}$ of sterile normal saline with the help of sterile syringe and placed in culture broth and vigorously stirred to release attached microorganisms of which $0.01 \mathrm{ml}$ was streaked onto blood agar and MacConkey agar, using a $4 \mathrm{~mm}$ inoculating loop and incubated at $37^{\circ} \mathrm{C}$ for 24 to 48 hours.

\section{Blood sampling and processing}

Blood $(10 \mathrm{ml})$ was collected from a peripheral vein (not through the catheter) within 48 hours of catheter collection under aseptic precautions in a blood culture bottle. After 24 hours subculture was made onto blood agar and Macconkey agar plates and incubated at $37^{\circ} \mathrm{C}$.In case of drainage from the catheter site,sample was taken with a sterile swab and inoculated onto blood agar and MacConkey agar plates and incubated at $37^{\circ} \mathrm{C}$. All the culture isolates are further identified by using standard biochemical tests up to genus/species level. Antibiotic sensitivity testing was done by the Modified Kirby-Bauer disc diffusion method according to the Clinical and Laboratory Standards Institute (CLSI) guidelines (2006).

\section{Detection of MRSA}

Screening for Methicillin-resistant Staphylococcus aureus (MRSA) was performed using an cefoxitin disk on Mueller Hinton agar.

\section{Detection of Extended spectrum Beta lactamase producers}

The screening for extended spectrum beta lactamase (ESBL) was done using, ceftazidime disc $(\leq 22 \mathrm{~mm})$ and cefotaxime $(\leq$ $27 \mathrm{~mm}$ ). The phenotypic confirmation was done by testing the strain against ceftazidime and ceftazidime with clavulanic acid discs. A $>5 \mathrm{~mm}$ diameter of the zone of inhibition for ceftazidime with clavulanic acid in comparison to ceftazidime was considered indicative of ESBL.

Catheter colonisation - significant growth of a microorganism on the catheter but no growth in blood cultures.

Local site infection - presence of erythema, swelling, tenderness, and purulent drainage around the catheter port and catheter tip shows significant growth of a microorganism on culture. Blood cultures may be positive or negative.

Significant growth in catheter tip was defined as $\geq 15$ colony forming units (CFU) by Maki's roll plate method or $\geq 10^{3} \mathrm{CFU} / \mathrm{ml}$ by the catheter flush method.

Criteria for CRBSI: Significant growth of catheter tip as well as the same organism (species and antibiogram) isolated from the catheter segment and peripheral blood culture.

\section{Results and Discussion}

Out of the 50 patients in the study group majority were in the $20-40$ year age group with a slight male preponderance. Out of the 50 patients studies 28 were admitted in the intensive care units. Among a total of 34 isolates, Coagulase negative staphylococci (44\%) were the most frequent isolates followed by Staphylococcus aureus (17.6\%) and Klebsiella pneumoniae (17.6\%). Diphtheroids and Pseudomonas constituted $14.7 \%$ and $5.8 \%$ respectively.

In this study, 18 patients had growth in the catheter tips (18 were positive by roll plate method out of which 8 were positive by flush method also).16 were blood culture positive. Similar isolates in catheter tip \& blood culture 
with the same antibiogram was observed among 5 patients (Table 1).

The incidence of CRBSI was calculated to be 1.38 per 1000 catheter days.(Deviceassociated infection rate $=$ Number of deviceassociated infections for an infection site $\mathrm{X}$ 1000 divided by Number of device days) (Table 2)

The antibiotic susceptibility pattern of the organisms are summarised in Figure-1

Out of the 6 Klebsiella isolates, 1 was an extended spectrum beta lactamase (ESBL) producer.. Among the 5 isolates which were implicated in CRBSI, 2 were MRCONS, 2 were MRSA and 1 was ESBL producing Klebsiella pneumoniae (Table 3).

Intravascular catheters are indispensable in current medical practice.. Although such catheters provide necessary vascular access, their use puts patients at risk of catheter related blood stream infections. The study was done at a tertiary care hospital over a period of 2 months to find the incidence of vascular catheter related blood stream infections among 50 adult patients. Twenty two of them were in the age group of 20-40 years and 13 were in the age group of 40-60 years. In the study by Ramanathan Parameswaran et al. the mean age in years among controls was 43.53 and that among patients with local catheter infections and CRBSI were 42.1 and 42.84 respectively. In this study, 28 patients were males and 22 were females. Maximum number of patients (28 patients) in the study were from the intensive care units and 22 patients were from wards. Maki DG et al in 2006 have reported that $32 \%$ of CRBSI were from the ICU (Maki et al., 2006).

All the patients included in the study had a mean duration of 10.5 days of hospital stay. They were catheterized for venous access to administer IV fluids and antibiotics. A majority of them (28 patients) especially in the ICU were catheterized in an emergency circumstance. 19 patients had diabetes, 7 had renal failure and 2 patients were on steroids. Ramanathan et al., have noted that the commonest premorbidity among the controls and patients with CRBSI was renal failure (35.5\% and $40 \%$ respectively) while that among the patients with local catheter infections was diabetes $(34.9 \%)$

In this study all the 50 catheters sampled were peripheral venous catheters made of Teflon. According to E. Bouza1 et al., Teflon or polyurethane catheters were associated with fewer infections than catheters made of PVC or polyethylene. All catheters were changed within 72 to 96 hours of insertion in this study. Eighteen patients in this study had growth in the catheter tips and 16 were blood culture positive. Similar isolates with the same antibiogram was observed among 5 patients (who also had clinical signs of infection) and those were considered as catheter related blood stream infections (CRBSI). Subba Rao et al., in their study reported that out of the 54 patients with positive tip cultures, 19 had corresponding positive blood cultures (Subba Rao et al., 2005). Only 7 patients had similar organisms grown from both the tip culture and simultaneous blood culture.

The incidence of CRBSI in this study was 1.38 per 1000 catheter days. It was calculated using the following formula.Deviceassociated infection rate $=$ Number of deviceassociated infections for an infection site $\mathrm{X}$ 1000 divided by Number of device days. Device-days are the total number of days of exposure to the device (e.g., vascular catheter) by all of the patients in the selected population during the selected time period.

The CRBSI rate of 1.38 per 1000 catheter days observed in this study is comparable to 
the study by Singh et al., (2010) in which the overall infection rate for CRBSI was found to be 0.48 per 1000 device days. According to Ramanathan et al., the incidence of CRBSI was 8.75 per 1,000 catheter days. Incidence of CRBSI reported varies from country to country and even hospital to hospitals.

Catheter tips were processed by Maki's roll plate method and flush method.18 had growth in the roll plate method, out of which 8 catheters had growth in the flush method also. Maki et al have considered Maki's semi quantitative method as the international diagnostic method. They have reported a specificity ranging between $76-96 \%$ for this method in diagnosing CRBSI. Twelve patients were found to have catheter colonisation in this study. Several studies have used significant catheter tip colonisation as a measure of CRBSI Local infections was found among 6 patients. All the 5 patients with CRBSI had local infections..

The low rates of CRBSI ( $p$ value 0.8) observed in this study may be due to the fact that all the catheters were changed within 7296 hours after insertion. This could also account for the low rates of local site infections. The low values obtained in this study have to be interpreted in the light of the small sample size studied and the multiple antibiotics received by the patient.

Thirty four isolates were obtained from the catheter tips and blood cultures. The majority of them were Coagulase negative staphylococci (44\%) followed by Staphylococcus aureus (17.6\%) and Klebsiella pneumoniae (17.6\%). Diphtheroids and Pseudomonas constituted $14.7 \%$ and 5.8 $\%$ respectively. In the study by Ramanathan parameswaran et al., $40 \%$ of the pathogens causing CRBSI was Staphylococcus aureus followed by Pseudomonas aeruginosa 16\%, CONS 8\% and Klebsiella pneumoniae 8\%.
Most cases of catheter related bloodstream infection are caused by skin commensals, These organisms migrate from the skin at the insertion site, and colonise the external catheter surface, eventually causing a catheter related bloodstream infection. This colonisation can take less than 24 hours following insertion. Microorganisms colonise the catheter by forming a bio film over the catheter surface Bacteria in bio films are more resistant to antimicrobials and host defence mechanisms

Approximately $65 \%$ of CRBSI originate from skin flora, $30 \%$ from contaminated hub and $5 \%$ from other pathways. Staphylococci are the most frequently isolated pathogens in catheter related infections particularly CONS, followed by Enterococci, Staphylococcus aureus and Candida spp. Microbial factors include bio film formation and adhesion of pathogens to catheter surfaces (CONS, staphylococcus aureus). In the study by Pittet et al., CONS, followed by Enterococci, Staphylococci aureus and Candida sp were the major pathogens observed. Before 1986, Staphylococcus aureus was the most frequently reported pathogen. The distribution of pathogens nevertheless varies with the type of patient, underlying condition, type of catheter, the quality of health care.

The antibiotic sensitivity pattern of the isolates showed that majority of CONS and Diphtheroids were sensitive to the drugs tested like Gentamicin, Cephalosporins, Ciprofloxacin, Amikacin, Ampicillin and Cotrimoxazole; Methicillin resistance was observed among 2 CONS and 2 Staphylococcus aureus isolates All the gram positive isolates were sensitive to vancomycin.Gram negative bacilli like Klebsiella pneumoniae and Pseudomonas sp showed a higher degree of resistance to Ciprofloxacin and Gentamicin; Most of them 
were sensitive to Amikacin, Ceftazidime and Ceftazidime with clavudinic acid.

Out of the 6 Klebsiella isolates, 1 was an extended spectrum beta lactamase (ESBL) producer. Among the 5 isolates which were implicated in CRBSI, 2 were MRCONS, 2 were $M R S A$ and 1 was ESBL producing K.pneumoniae. In a study in Brazil by Sadoyma et al., $41 \%$ of the isolates were MRSA and 59\% were Methicillin sensitive.

Table.1 Catheter Tip and Blood Culture Results

\begin{tabular}{|c|c|c|c|c|}
\hline \multirow[t]{2}{*}{ Isolates } & \multicolumn{2}{|c|}{ 1.Catheter tip } & \multirow{2}{*}{$\begin{array}{c}\text { 2.Blood } \\
\text { culture } \\
n=16\end{array}$} & \multirow{2}{*}{$\begin{array}{c}\text { CRBSI } \\
\text { (similar isolates } \\
\text { in } 1 \& 2 \text { ) } n=5\end{array}$} \\
\hline & $\begin{array}{c}\text { Roll plate } \\
\text { method } n=18\end{array}$ & $\begin{array}{c}\text { Flush method } \\
n=8\end{array}$ & & \\
\hline CONS & 10 & 4 & 5 & 2 \\
\hline Diphtheroids & 2 & 1 & 3 & - \\
\hline Staphylococcus aureus & 3 & 2 & 3 & 2 \\
\hline Klebsiella pneumoniae & 3 & 1 & 3 & 1 \\
\hline Pseudomonas spp & - & - & 2 & - \\
\hline
\end{tabular}

Table.2 Incidence of Catheter Related Infections

\begin{tabular}{|l|c|c|}
\hline \multicolumn{1}{|c|}{ Results } & Number & Percentage \\
\hline Catheter colonisation & 12 & $24 \%$ \\
\hline Local catheter site infection & 6 & $12 \%$ \\
\hline CRBSI & 5 & $10 \%$ \\
\hline
\end{tabular}

Table.3 Prevalence of MRSA \& ESBL among the isolates

\begin{tabular}{|l|c|}
\hline \multicolumn{1}{|c|}{ Resistant isolates } & Number \\
\hline$M R C O N S$ & 2 \\
\hline$M R S A$ & 2 \\
\hline ESBL Klebsiella pneumoniae & 1 \\
\hline
\end{tabular}

Fig.1 Antibiotic Susceptibility Patterns of the Isolates

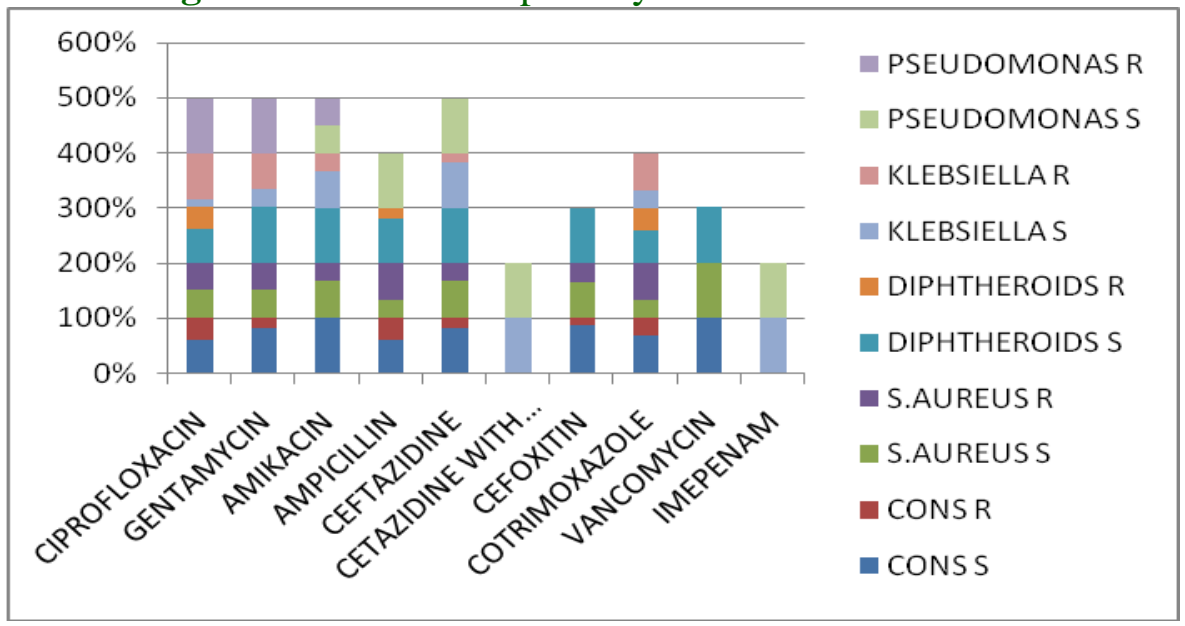


Among the MRSA isolates, multiple drug resistance to Gentamicin, Cotrimethoxazole, Erythromycin was seen. Knowledge of the CRBSI in a particular centre is the first-step toward assessing the success of existing measures to prevent these infections and the need for further corrective measures. Using proper aseptic technique, changing catheters at a fixed interval and using catheters made of materials more resistant to adherence by pathogens can reduce the number of bloodstream infections at a facility.

In conclusion, CRBSI is an iatrogenic problem that causes significant morbidity and mortality, excess length of hospital stay and excess costs of health care especially to patients in the ICUs. An estimation of the rate of infection, and identification of the causative organisms will provide a clue as to the route of entry and pathogenesis of the infection, and this in turn may point to effective preventive strategies. The findings from this study will help to implement educational programs on CRBSI for health personnel and help to initiate infection control interventions at the earliest.

\section{References}

Anaissie, E., Samonis, G., Kontoyiannis, D., et al. 1995. Role of catheter colonization and infrequent haematogenous seeding in catheterrelated infections. Eur. J. Clin. Microbiol. Infect. Dis., 14(2): 134-7.

Bjornson, H.S. 1993. Pathogenesis, prevention, and management of catheter-associated infections. New Horiz., 1(2): 271-8.

Bjornson, H.S., Colley, R., Bower, R.H., Duty, V.P., Schwartz-Fulton, J.T., Fischer, J.E. 1982. Association between microorganism growth at the catheter insertion site and colonization of the catheter in patients receiving total parenteral nutrition. Surgery, (4): 720-7.
Bouza, E., Burillo, A., Muñoz, P. 2002. Catheter-related infections: diagnosis and intravascular treatment. Clin. Microbiol. Infect., 8(5): 265-274.

Clinical and Laboratory Standards Institute. 2006. Performance standards for antimicrobial susceptibility testing; 16th informational supplement. M100-S16. Clinical and Laboratory Standards Institute, Wayne.

Geraldo, S., Augusto, D.F. 2006. Central Venous Catheter-Related Bloodstream Infection Caused by Staphylococcus aureus: Microbiology and Risk Factors. The Brazilian J. Infect. Dis., 10(2): 100106.

Kimura, A.C., Calvet, H., Higa, J.I., et al. 2005. Outbreak of Ralstonia pickettii bacteraemia in a neonatal intensive care unit. Pediatr. Infect. Dis. J., 24(12):1099-103.

Leonidia, L., Charalambos A. Gogos. Catheter-related bloodstream infections: catheter management according to pathogen. International Journal of Antimicrobial Agents, Elsevier, 2010, 36.

Maki, D.G., Kluger, D.M., Crnich, C.J. 2006. The risk of bloodstream infection in adults with different intravascular devices: a systematic review of 200 published prospective studies. Mayo Clin. Proc., 81(9): 1159-1171.

Maki, D.G., Weise, C.E., Sarafin, H.W. 1977. A semi quantitative culture method for identifying intravenous catheter-related infections. N Engl. J. Med., 296: 13051309.

Pittet, D., Rangel-Frausto, S., Li, N., et al. 1995. Systemic inflammatory response syndrome, sepsis, severe sepsis and septic shock: incidence, morbidities and outcomes in surgical ICU patients. Intensive Care Med., 21(4): 302-9.

Ramanathan, P., Jatan, B., Sherchan, M., Varma, D., Chiranjay, M., Sudha, V. 
2011. Intravascular catheter-related infections in an Indian tertiary care hospital. J. Infect. Dev. Ctries., 5(6): 452-458.

Rijnders, B.E., Van, W., Peetermans, W. Catheter-tip colonization as a surrogate end point in clinical studies on catheterrelated bloodstream infection: how strong is the evidence? Clin. Infect. Dis., 35(9):1053-8.

Subba Rao, S.D., Joseph, M.P., Lavi, R., Macaden, R. 2005. Infections related to vascular catheters in a pediatric intensive care unit. Indian Pediatrics, 42(7): 667-672.

Vincent, J.L., Bihari, D.J., Suter, P.M., et al. 1995. The prevalence of nosocomial infection in intensive care units in Europe. Results of the European Prevalence of Infection in Intensive Care (EPIC) Study. EPIC International Advisory Committee. Jama, 274(8): $639-44$.

\section{How to cite this article:}

Sainivedeta, M.V.M., I.M. Rejitha, G. Sucilathangam and Revathy, C. 2017. Vascular Catheter Related Infections in a Tertiary Care Hospital - A Microbiological Study. Int.J.Curr.Microbiol.App.Sci. 6(1): 592-599. doi: http://dx.doi.org/10.20546/ijcmas.2017.601.072 